\title{
Anticipating integrals and martingales on the Poisson space
}

\author{
Giovanni Peccati ${ }^{1}$ Ciprian A. Tudor ${ }^{2}$ \\ ${ }^{1}$ Laboratoire de Statistique Théorique et Appliquée, Université de Paris 6, \\ 4, place Jussieu, F-75252 Paris Cedex 05, France. \\ giovanni.peccati@gmail.com \\ ${ }^{2}$ SAMOS/MATISSE, Université de Panthéon-Sorbonne Paris 1, \\ 90, rue de Tolbiac, F-75634 Paris Cedex 13, France. \\ tudor@univ-paris1.fr
}

July 19, 2018

\begin{abstract}
Let $\tilde{N}_{t}$ be a standard compensated Poisson process on $[0,1]$. We prove a new characterization of anticipating integrals of the Skorohod type with respect to $\tilde{N}$, and use it to obtain several counterparts to well established properties of semimartingale stochastic integrals. In particular we show that, if the integrand is sufficiently regular, anticipating Skorohod integral processes with respect to $\tilde{N}$ admit a pointwise representation as usual Itô integrals in an independently enlarged filtration. We apply such a result to: (i) characterize Skorohod integral processes in terms of products of backward and forward Poisson martingales, (ii) develop a new Itôtype calculus for anticipating integrals on the Poisson space, and (iii) write Burkholder-type inequalities for Skorohod integrals.
\end{abstract}

Key words: Poisson processes; Malliavin Calculus; Skorohod integrals; Itô formula; Burkholder inequalities.

AMS 2000 Classification: 60G51; 60H05; 60H07

\section{Introduction}

Let $\tilde{N}_{t}$ be a standard compensated Poisson process on $[0,1]$. The aim of this paper is to prove a new characterization of anticipating integrals (of the Skorohod type) with respect to $\tilde{N}$, and to apply such a result to investigate the relations between anticipating integrals and Poissonian martingales.

The anticipating Skorohod integral has been first introduced in [25] in the context of Gaussian processes. It is well known that the notion of Skorohod integral can be naturally extended to the family of normal martingales, that is, martingales having a conditional quadratic variation equal to $t$ (among which there are the Wiener process and the compensated Poisson process). In this case, the Skorohod integral is an extension of the classical (semimartingale) Itô integral to a wider family of non-adapted integrands, and therefore coincides with the latter on the class of (square integrable) adapted processes. See, for instance, [10; we also refer to [12] for an exhaustive presentation of results, techniques and applications of the anticipating stochastic calculus in the Gaussian context. In this paper, we try to deal with some of the disadvantages of the Skorohod calculus, in the specific 
case of the compensated Poisson process, and from the standpoint of the classic semimartingale theory.

To better understand our motivations, consider a Skorohod integral process (see [10 for the precise setup) with the form

$$
X_{t}=\int_{0}^{t} u_{s} \delta Z_{s} \quad t \in[0,1]
$$

where $Z$ is a normal martingale and $u \mathbf{1}_{[0, t]}$ belongs to the domain of the Skorohod integral $\delta, \forall t$. Then, in general, if the integrand $u$ is not adapted to the natural filtration of $Z$, the process $X$ is not a semimartingale, and the study of $X$ cannot be carried out by means of the usual Itô theory (as presented for instance in [5]). Actually, the techniques employed to deal with processes such as $X$ mostly stem from functional analysis, and they do not allow, e.g., to obtain fine trajectorial properties. However, when $Z=W$, where $W$ stands for a standard Wiener process, the authors of the present paper have pointed out several remarkable connections between processes such as $X$ in (11) and Wiener martingales. In particular, the following results (among others) have been obtained when $Z=W$ :

i) the class of Skorohod integral processes with sufficiently regular integrands coincides with a special family of Itô integrals, called Itô-Skorohod integrals (see [28]);

ii) a Skorohod integral process $X$ such as (11) can be approximated, in a certain norm, by linear combinations of processes with the form $M_{t} \times M_{t}^{\prime}$, where $M_{t}$ is (centered) Wiener martingale and $M_{t}^{\prime}$ is a backward Wiener martingale (see [18]).

These facts lead in a natural way to explore the anticipating integrals in the context of a standard Poisson process. It is known that a Skorohod type integration can be developed on the Poisson space by using the Fock space structure, and that such integrals enjoy a number of useful properties, in part analogous to the ones displayed by Skorohod operators on Wiener space. We refer e.g. to [14, [16], 3], 9], 20, 21] or [10] for different aspects of this calculus. Here, we shall provide Poissonian counterparts to several results given in [28] and [18, as facts (i) and (ii) above, and we shall systematically point out the arguments that differ from those given in the Wiener context. We remark that some of our results are of particular interest in the Poisson case. For example, our methods allow to obtain an Itô-type formula for anticipating integrals, and - as far as we know - this is the only anticipating change of variables formula for the Poisson situation (we could not find, for instance, an Itô-type formula proved in the spirit of [13]). We note that the fact that the increments of the Poisson process are independent plays a crucial role in our construction; therefore the extension of the results to a more general normal martingale seems difficult.

The paper is organized as follows. The first part of Section 2 contains some preliminaries on the Malliavin calculus with respect to the Poisson process, whereas the second part displays a discussion about Itô stochastic integrals, $\sigma$-fields and (independently enlarged) filtrations on the Poisson space; we shall note that most of the results given here are still valid on the Wiener space. In Section 3, we show that every anticipating (Poisson) integral coincides pointwise with a special type of Itô integral and (as in [18]) we apply this relation to approximate Skorohod integral processes by linear combinations of processes that are a product of forward and backward martingales. Finally, in Section 4 we develop a new stochastic calculus of the Itô type for anticipating Poisson integrals. 


\section{Preliminaries: Malliavin calculus and filtrations}

\subsection{Malliavin calculus for the Poisson process}

Throughout the paper, we use notation and terminology from standard semimartingale theory. The reader is referred e.g. to [5], 4] or [23] for any unexplained notion.

Let $T=[0,1]$ and let $N=\left(N_{t}\right)_{t \in T}$ be a standard Poisson process, defined on the standard Poisson space $(\Omega, \mathbb{F}, \mathbf{P})$ (see e.g. 14]). By $\tilde{N}$ we will denote the compensated Poisson process $\tilde{N}_{t}=N_{t}-t$. For every Borel set $B$, we will note $N(B)$ and $\tilde{N}(B)$, respectively, the random measures $\sum_{s \in B} \Delta N_{s}$ and $N(B)-\lambda(B)$, where $\Delta N_{s}=N_{s}-N_{s-}$ and $\lambda$ stands for Lebesgue measure on $T$. It is well known (see e.g. [4] or [10]) that the process $t \mapsto \tilde{N}_{t}$ is a normal martingale, that is, $\tilde{N}_{t}$ is a càdlàg martingale initialized at zero, such that its conditional quadratic variation process (or angle bracket process) is given by $\langle\tilde{N}, \tilde{N}\rangle_{t}=\langle\tilde{N}\rangle_{t}=t$. The quadratic variation process of $\tilde{N}$ (or right bracket process) is of course $[\tilde{N}, \tilde{N}]_{t}=[\tilde{N}]_{t}=N_{t}$ (note that the results of our paper extend immediately to the case of a Poisson process on $\mathbb{R}_{+}$, with a deterministic intensity $\mu>0$ ). It is also known (see again [10] and the references therein) that $\tilde{N}$ enjoys the chaotic representation property, i.e. every random variable $F \in L^{2}(\Omega, \mathbb{F}, \mathbf{P})=L^{2}(\mathbf{P})$, measurable with respect to the $\sigma$-algebra generated by $N$, can be written as an orthogonal sum of multiple Poisson-Itô stochastic integrals

$$
F=\mathbf{E}(F)+\sum_{n \geq 1} I_{n}\left(f_{n}\right)
$$

where the infinite series converges in $L^{2}(\mathbf{P})$, and, for $n \geq 1$, the kernel $f_{n}$ is an element of $L_{s}^{2}\left(T^{n}\right) \subset$ $L^{2}\left(T^{n}\right)$, where $L_{s}^{2}\left(T^{n}\right)$ and $L^{2}\left(T^{n}\right)$ denote, respectively, the space of symmetric and square integrable functions, and the space of square integrable functions on $T^{n}$ (endowed with Lebesgue measure).

Let us recall the basic construction of the multiple Poisson-Itô integral on the Poisson space. Fix $n \geq 2$ and denote by $S_{n}$ and $\widetilde{S}_{n}$, respectively, the vector space generated by simple functions with the form

$$
f\left(t_{1}, \ldots, t_{n}\right)=\mathbf{1}_{B_{1}}\left(t_{1}\right) \ldots \mathbf{1}_{B_{n}}\left(t_{n}\right),
$$

where $B_{1}, \ldots, B_{n}$ are disjoint subsets of $\mathbb{R}$, and the vector space generated by the symmetrization of the element of $S_{n}$. If $f$ is as in (3) and $\tilde{f} \in \widetilde{S}_{n}$ is its symmetrization, we define $I_{n}(\tilde{f})$ as

$$
I_{n}(\tilde{f})=\tilde{N}\left(B_{1}\right) \ldots \tilde{N}\left(B_{n}\right)
$$

Since, for every $n \geq 2, \widetilde{S}_{n}$ is dense in $L_{s}^{2}\left(T^{n}\right)$, the integral $I_{n}$ can be extended to $L_{s}^{2}\left(T^{n}\right)$ by continuity, due to the isometry formula, true for every $m, n \geq 2, \tilde{f} \in \widetilde{S}_{n}$ and $\tilde{g} \in \widetilde{S}_{m}$,

$$
\mathbf{E}\left(I_{n}(\tilde{f}) I_{m}(\tilde{g})\right)=n !\langle\tilde{f}, \tilde{g}\rangle_{L^{2}\left(T^{n}\right)} \mathbf{1}_{(n=m)} .
$$

We also use the following conventional notation: $L^{2}(T)=L^{2}\left(T^{1}\right)=L_{s}^{2}\left(T^{1}\right) ; I_{1}(f)=\int_{0}^{1} f(s) d \tilde{N}_{s}$, $f \in L^{2}(T) ; \tilde{f}$ is the symmetrization of $f, \forall f \in L^{2}\left(T^{n}\right), n \geq 2 ; I_{n}(f)=I_{n}(\tilde{f}), f \in L^{2}\left(T^{n}\right), n \geq 2$; $L^{2}\left(T^{0}\right)=L_{s}^{2}\left(T^{0}\right)=S_{0}=\widetilde{S}_{0}=\mathbb{R} ; I_{0}(c)=c, c \in \mathbb{R}$. 
Remark 1 As proved e.g. by Ogura in [17], one can define multiple stochastic integrals on the Poisson space by using the Charlier-Poisson polynomials. More precisely, for $n \geq 0$, the $n$th CharlierPoisson polynomial $C_{n}(t, x),(t, x) \in[0,1] \times \mathbb{R}$, is defined through the generating function (see for instance [Q])

$$
\Phi(z, t, x)=\sum_{n=0}^{\infty} z^{n} C_{n}(t, x)=(1+z)^{x+t} \exp (-z t) .
$$

It is well known (see e.g. [7, Lemma 2]) that the Charlier polynomials are connected to the above defined Poisson-Itô multiple integrals by the following relation: for every Borel subset $B \subseteq T$

$$
C_{n}(\lambda(B), \tilde{N}(B))=\frac{1}{n !} I_{n}\left(1_{B}^{\otimes n}(\cdot)\right),
$$

where $n \geq 1$, and $\lambda$ stands for Lebesgue measure.

Now define, for $n, m \geq 1, f \in L_{s}^{2}\left(T^{m}\right), g \in L_{s}^{2}\left(T^{n}\right), r=0, \ldots, m \wedge n$ and $l=1, \ldots, r$, the (contraction) kernel on $T^{m+n-r-l}$

$$
\begin{aligned}
& f \star_{r}^{l}\left(\gamma_{1}, \ldots, \gamma_{r-l}, t_{1}, \ldots, t_{m-r}, s_{1}, \ldots, s_{n-r}\right) \\
= & \int_{T^{l}} f\left(u_{1}, \ldots, u_{l}, \gamma_{1}, \ldots, \gamma_{r-l}, t_{1}, \ldots, t_{m-r}\right) g\left(u_{1}, \ldots, u_{l}, \gamma_{1}, \ldots, \gamma_{r-l}, s_{1}, \ldots, s_{n-r}\right) d u_{1} \ldots d u_{l},
\end{aligned}
$$

and, for $l=0$,

$$
f \star_{r}^{0}\left(\gamma_{1}, \ldots, \gamma_{r}, t_{1}, \ldots, t_{m-r}, s_{1}, \ldots, s_{n-r}\right)=f\left(\gamma_{1}, \ldots, \gamma_{r}, t_{1}, \ldots, t_{m-r}\right) g\left(\gamma_{1}, \ldots, \gamma_{r}, s_{1}, \ldots, s_{n-r}\right),
$$

so that

$$
f \star_{0}^{0}\left(t_{1}, \ldots, t_{m}, s_{1}, \ldots, s_{n}\right)=f\left(t_{1}, \ldots, t_{m}\right) g\left(s_{1}, \ldots, s_{n}\right) .
$$

We will need the following product formula for two Poisson-Itô multiple integrals (see [7, 22], or [26]): let $f \in L_{s}^{2}\left(T^{m}\right)$ and $g \in L_{s}^{2}\left(T^{n}\right), n, m \geq 1$, and suppose moreover that $f \star_{r}^{l} g \in L^{2}\left(T^{m+n-r-l}\right)$ for every $r=0, \ldots, m \wedge n$ and $l=1, \ldots, r$, then

$$
I_{m}(f) I_{n}(g)=\sum_{r=0}^{m \wedge n} r !\left(\begin{array}{c}
m \\
r
\end{array}\right)\left(\begin{array}{l}
n \\
r
\end{array}\right) \sum_{l=0}^{r} I_{m+n-r-l}\left(f \star_{r}^{l} g\right) .
$$

It is possible to develop a Malliavin-type calculus with respect to the Poisson process based on the (symmetric) Fock space isomorphism induced by formulae (2) and (5). We refer to [14] or [10] for the basic elements of this calculus. For a random variable $F$ as in (2) we introduce the annihilation (or derivative) operator as

$$
D_{t} F=\sum_{n \geq 1} n I_{n-1}\left(f_{n}(\cdot, t)\right), \quad t \in T
$$

and its domain, usually denoted by $\mathbb{D}^{1,2}$, is the set

$$
\mathbb{D}^{1,2}=\left\{F=\sum_{n \geq 0} I_{n}\left(f_{n}\right): \sum_{n} n n !\left\|f_{n}\right\|_{n}^{2}<+\infty\right\}
$$


where $\|\cdot\|_{n}$ is the norm in $L^{2}\left(T^{n}\right)$. The operator $D$ is not a derivation (see [10, p. 91]) and it satisfies (see [14, Lemma 6.1 and Theorem 6.2])

$$
D(F G)=F D G+G D F+D F D G \text { if } F, G, F G \in \mathbb{D}^{1,2} .
$$

The Skorohod integral, or the creation operator, is defined by

$$
\delta(u)=\sum_{n \geq 0} I_{n+1}\left(\tilde{f}_{n}\right)
$$

whenever $u_{t}=\sum_{n \geq 0} I_{n}\left(f_{n}(\cdot, t)\right)$, where $u \in L^{2}(T \times \Omega)$, belongs to the domain of $\delta$, noted $\operatorname{Dom}(\delta)$, that is, $u$ verifies

$$
\sum_{n \geq 0} n n !\left\|f_{n}\right\|_{n+1}^{2}<+\infty
$$

We introduce the subset $\mathbb{L}^{1,2}$ of $\operatorname{Dom}(\delta)$ defined as

$$
\mathbb{L}^{1,2}=\left\{u_{t}=\sum_{n} I_{n}\left(f_{n}(\cdot, t)\right): \sum_{n}(n+1) !\left\|f_{n}\right\|_{n+1}^{2}<+\infty\right\} .
$$

Note that $\mathbb{L}^{1,2}$ equals $L^{2}\left(T ; \mathbb{D}^{1,2}\right)$, when the former is endowed with the seminorm

$$
\|u\|_{1,2}^{2}=\mathbf{E} \int_{0}^{1} u_{s}^{2} d s+\mathbf{E} \int_{0}^{1} \int_{0}^{1}\left(D_{r} u_{s}\right)^{2} d r d s
$$

and moreover, for every $u \in \mathbb{L}^{1,2}$, one can verify the inequality

$$
\mathbf{E} \delta(u)^{2} \leq\|u\|_{1,2}^{2}
$$

(see for instance formula (13) below). For any integer $k \geq 2$ the space $\mathbb{D}^{k, 2}$ denotes the set of $k$ times weakly differentiable random variables, endowed with the seminorm

$$
\|F\|_{k, 2}^{2}=\mathbf{E}\|F\|^{2}+\sum_{l=1}^{k}\left\|D^{l} F\right\|_{L^{2}\left(T^{l} \times \Omega\right)}^{2}, \quad F \in \mathbb{D}^{k, 2},
$$

where $D^{1}=D$, and, for $l \geq 2$, the $l$ th Malliavin derivative $D^{l}$ is first defined by iteration on simple functionals, and then extended by a standard closure procedure. By $\mathbb{L}^{k, 2}$ we denote the set $L^{2}\left(T ; \mathbb{D}^{k, 2}\right)$; note that, if $u \in \mathbb{L}^{1,2}$, then $u \mathbf{1}_{[0, t]} \in \operatorname{Dom}(\delta)$ for every $t \in T$ (see [10, Lemma 4.6]). Occasionally, we will also use the notation

$$
\int_{0}^{1} u_{s} \delta \tilde{N}_{s}=\delta(u), \quad u \in \operatorname{Dom}(\delta)
$$

We recall that, according e.g. to [10, Proposition 4.4], if $u \in L^{2}(T \times \Omega)$ is a predictable process with respect to the filtration generated by $\tilde{N}$, then $u$ is Skorohod integrable and $\delta(u)$ coincides with the usual Itô integral with respect to the càdlàg martingale $\tilde{N}$.

The following duality relationship between $D$ and $\delta$, which is classic in the Gaussian case, still holds on the Poisson space (see [14, Proposition 4.2])

$$
\mathbf{E}\left[\int_{0}^{1}\left(D_{s} F u_{s}\right) d s\right]=\mathbf{E}(\delta(u) F) \text { if } F \in \mathbb{D}^{1,2} \text { and } u \in \operatorname{Dom}(\delta) .
$$


and the covariance of two Skorohod integrals is given by

$$
\mathbf{E}(\delta(u) \delta(v))=\mathbf{E} \int_{0}^{1} u_{s} v_{s} d s+\mathbf{E} \int_{0}^{1} \int_{0}^{1} D_{r} u_{s} D_{s} v_{r} d r d s
$$

whenever $u, v \in \mathbb{L}^{1,2}$ (see [14, Thorem 4.1]). Eventually, we will need the commutativity relationship between $D$ and $\delta$

$$
D_{t} \delta(u)=u_{t}+\delta\left(D_{t} u\right) \text { if } u \in \mathbb{L}^{1,2} \text { and } D_{t} u \in \operatorname{Dom}(\delta)
$$

(see 14. Thorem 4.1]), as well as the integration by parts formula, true whenever $F \in \mathbb{D}^{1,2}$ and $D F \times u \in \operatorname{Dom}(\delta)$,

$$
\delta(F u)=F \delta(u)-\langle D F, u\rangle_{L^{2}(T)}-\delta(D F u)
$$

(see [14, Theorem 7.1]).

\section{$2.2 \sigma$-fields, filtrations and Itô stochastic integrals}

For any Borel set $A \subseteq T$, we use the notation

$$
\mathbb{F}_{A}=\sigma\left\{\tilde{N}\left(h \mathbf{1}_{A}\right): h \in L^{2}(T)\right\}
$$

and also, for $t \in T, \mathbb{F}_{t}=\mathbb{F}_{[0, t]}$ and $\mathbb{F}_{t^{c}}=\mathbb{F}_{[0, t]^{c}}$. Note that, in the following, we will tacitly complete each $\sigma$-field $\mathbb{F}_{A}$ with the $\mathbf{P}$-null sets of $\mathbb{F}$, so that, for instance, $\left\{\mathbb{F}_{t}: t \in T\right\}$ is the completion of the natural filtration of the process $\tilde{N}_{t}$. We also set $\mathbb{F}=\mathbb{F}_{1}$. The independence of the Poisson increments implies the following relations (see again [14]): for every $n \geq 1$, every $f \in L_{s}^{2}\left(T^{n}\right)$ and every Borel subset $A$ of $T$,

$$
\mathbf{E}\left(I_{n}(f) \mid \mathbb{F}_{A}\right)=I_{n}\left(f \mathbf{1}_{A}^{\otimes n}\right) \text {, a.s. }-\mathbf{P},
$$

and, a.s. $-\mathbf{P}$

$$
D_{t} \mathbf{E}\left(F \mid \mathbb{F}_{A}\right)=\mathbf{E}\left(D_{t} F \mid \mathbb{F}_{A}\right) \mathbf{1}_{A}(t), \quad t \in T .
$$

An immediate consequence of (17) is that, if $F \in \mathbb{D}^{1,2}$ is a $\mathbb{F}_{A}$-measurable random variable, then $D F=0$ on $A^{c} \times \Omega$. Moreover, if we denote by $X$ the Skorohod integral process

$$
X_{t}=\int_{0}^{t} u_{s} \delta \tilde{N}_{s}=\delta\left(u \mathbf{1}_{[0, t]}\right), \quad t \in T, u \in \mathbb{L}^{1,2},
$$

then the process $X$ satisfies (see Lemma 3.2.1 in [12])

$$
\mathbf{E}\left(X_{t}-X_{s} \mid \mathbb{F}_{[s, t]^{c}}\right)=0 \text { for every } s<t .
$$

In the anticipating calculus, relation (18) plays roughly the same role as does, in the usual Itô calculus, the martingale characterization of adapted stochastic integrals. filtration

Now fix $t \in(0,1]$. In the sequel, we will use the properties of the following (enlarged)

$$
\mathbb{F}_{(\cdot, t]^{c}}=\left\{\mathbb{F}_{(s, t]^{c}}: s \in[0, t]\right\}=\left\{\mathbb{F}_{s} \vee \mathbb{F}_{t^{c}}: s \in[0, t]\right\} .
$$

Note that, since $\tilde{N}$ has càdlàg paths, $\mathbb{F}_{(s, t]^{c}}=\mathbb{F}_{(s, t)^{c}}$ for every $s \in[0, t]$, and also

$$
\mathbb{F}_{[s, t]^{c}}=\bigvee_{\varepsilon>0} \mathbb{F}_{(s-\varepsilon, t]^{c}} .
$$


It is also easily checked that the filtration $\mathbb{F}_{(\cdot, t]^{c}}$ satisfies the usual conditions. We can therefore define, for every $t \in(0,1],{ }^{(p, t)}(\cdot)$ to be the predictable projection operator with respect to $\mathbb{F}_{(\cdot, t]^{c}}$, as defined e.g. in [5, Theorem 6.39]. Note that, due to the independence of its increments, the process $\tilde{N}_{r}, r \in[0, t]$, is again a normal martingale with respect to the filtration $\mathbb{F}_{(r, t]^{c}}$. It follows that the Itô (semimartingale) stochastic integral of a square integrable, $\mathbb{F}_{(\cdot, t]^{c}}$ - predictable process is always well defined. For a process $u \in L^{2}(T \times \Omega)$, whose restriction to $[0, t]$ is also $\mathbb{F}_{(\cdot, t]^{c}}$ - predictable, we will note $\int_{0}^{t} u_{s} d \tilde{N}_{s}$ the Itô stochastic integral of $u$ with respect to $\tilde{N}$, regarded as a càdlàg, square integrable $\mathbb{F}_{(\cdot, t]^{c}}$ - martingale on $[0, t]$. Note that we write $\int_{0}^{t}$ instead of $\int_{0+}^{t}$, because $\tilde{N}_{0}=0$. The

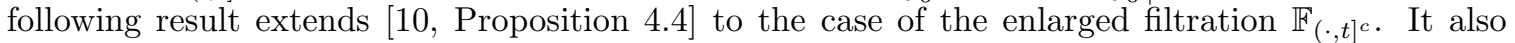
contains a Clark-Ocone type formula (see e.g. [16 for the Brownian case) which will be further generalized in the next section.

Proposition 1 Let the above notation prevail and fix $t \in(0,1]$. Then,

(i) if the restriction to $[0, t]$ of a process $u \in L^{2}(T \times \Omega)$ is predictable with respect to the filtration $\mathbb{F}_{(\cdot, t]^{c}}$, then

$$
u \mathbf{1}_{[0, t]} \in \operatorname{Dom}(\delta) \quad \text { and } \delta\left(u \mathbf{1}_{[0, t]}\right)=\int_{0}^{t} u_{s} d \tilde{N}_{s},
$$

where the right-hand side is a stochastic integral in the semimartingale sense;

(ii) for every $\mathbb{F}_{t}$-measurable functional $G \in \mathbb{D}^{1,2}$, and for every $0 \leq s \leq t$,

$$
G=\mathbf{E}\left(G \mid \mathbb{F}_{(s, t]^{c}}\right)+\delta\left({ }^{(p, t)}(D . G)\right)=\mathbf{E}\left(G \mid \mathbb{F}_{(s, t]^{c}}\right)+\int_{s}^{t}{ }^{(p, t)}\left(D_{r} G\right) d \tilde{N}_{r} .
$$

Proof. (i) Fix $t \in(0,1]$. We start by considering a process with the form

$$
u_{s}=g(s) I_{n}\left(h^{\otimes n} \mathbf{1}_{[0, s) \cup(t, 1]}^{\otimes n}\right), \quad s \in T,
$$

where $n \geq 1, h^{\otimes n}\left(t_{1}, \ldots, t_{n}\right)=h\left(t_{1}\right) \cdots h\left(t_{n}\right)$, and $h, g \in L^{2}(T)$. Plainly, $u \mathbf{1}_{[0, t]} \in \operatorname{Dom}(\delta)$. Now define, for $k=0, \ldots, n$ and $s<t$

$$
B_{k}^{n}(s)=\left\{\left(t_{1}, \ldots, t_{n}\right) \in T^{n}: k \text { of the } t_{i} \text { 's are }<s \text { and } n-k \text { are }>t\right\} .
$$

Of course,

$$
u_{s}=\sum_{k=0}^{n} u_{s}^{k}, \quad s \in T
$$

where, for $k=0, \ldots, n$,

$$
u_{s}^{k}=g(s) I_{n}\left(h^{\otimes n} \mathbf{1}_{B_{k}^{n}(s)}\right)=\left(\begin{array}{l}
n \\
k
\end{array}\right) g(s) I_{k}\left(h^{\otimes k} \mathbf{1}_{[0, s)}^{\otimes k}\right) I_{n-k}\left(h^{\otimes n-k} \mathbf{1}_{(t, 1]}^{\otimes n-k}\right),
$$

with $I_{0}=1$, the last equality being justified by an application of (7). Now fix $k$, and observe that the process $u_{s}^{k}, s \in[0, t]$, is predictable with respect to $\mathbb{F}_{(\cdot, t]^{c}}$ so that, on $[0, t]$, the Itô integral of $u^{k}$ with respect to $\tilde{N}$ is classically given by

$$
\int_{0}^{t} u_{s}^{k} d \tilde{N}_{s}=\left(\begin{array}{l}
n \\
k
\end{array}\right) I_{n-k}\left(h^{\otimes n-k} \mathbf{1}_{(t, 1]}^{\otimes n-k}\right) \int_{0}^{t} g(s) I_{k}\left(h^{\otimes k} \mathbf{1}_{[0, s)}^{\otimes k}\right) d \tilde{N}_{s} .
$$


On the other hand, the symmetrization in the $n+1$ variables $\left(t_{1}, t_{2}, \ldots, t_{n+1}\right)$ of the function $g \mathbf{1}_{[0, t]}\left(t_{1}\right)$ $\times h^{\otimes n} \mathbf{1}_{B_{k}^{n}\left(t_{1}\right)}\left(t_{2}, \ldots, t_{n+1}\right)$ is

$$
f_{n+1}^{k}\left(t_{1}, \ldots, t_{n+1}\right)=\frac{1}{n+1} \sum_{i=1}^{n+1} g \mathbf{1}_{[0, t]}\left(t_{i}\right) h^{\otimes n} \mathbf{1}_{B_{k}^{n}\left(t_{i}\right)}\left(t_{j}: j \neq i\right),
$$

and the restriction of $f_{n+1}^{k}$ to $\Delta^{n+1}=\left\{\left(t_{1}, \ldots, t_{n+1}\right) \in T^{n+1}: 0<t_{1}<\ldots<t_{n+1}<1\right\}$ is therefore

$$
\begin{aligned}
& \frac{1}{n+1} g \mathbf{1}_{[0, t]}\left(t_{k+1}\right) h^{\otimes n}\left(t_{1}, \ldots, t_{k}, t_{k+2}, \ldots, t_{n+1}\right) \mathbf{1}_{\left\{t_{k+2}>t\right\}} \\
= & \frac{1}{n+1} g \mathbf{1}_{[0, t]}\left(t_{k+1}\right) h^{\otimes k}\left(t_{1}, \ldots, t_{k}\right) h^{\otimes n-k}\left(t_{k+2}, \ldots, t_{n+1}\right) \mathbf{1}_{\left\{t_{k+2}>t\right\}}, \quad\left(t_{1}, \ldots, t_{n+1}\right) \in \Delta^{n+1}
\end{aligned}
$$

and consequently

$$
\begin{aligned}
\delta\left(u^{k}\right)= & n ! \int_{t}^{1} \int_{t}^{t_{n+1}} \cdots \int_{t}^{t_{k+3}} h^{\otimes n-k}\left(t_{k+2}, \ldots, t_{n+1}\right) d \tilde{N}_{t_{k+2}} d \tilde{N}_{t_{k+3}} \ldots d \tilde{N}_{t_{n+1}} \times \\
& \times \int_{0}^{t} g\left(t_{k+1}\right) \int_{0}^{t_{k+1}} \cdots \int_{0}^{t_{2}} h^{\otimes k}\left(t_{1}, \ldots t_{k}\right) d \tilde{N}_{t_{1}} \cdots d \tilde{N}_{t_{k}} d \tilde{N}_{t_{k+1}} \\
= & \left(\begin{array}{c}
n \\
k
\end{array}\right) I_{n-k}\left(h^{\otimes n-k} \mathbf{1}_{(t, 1]}^{\otimes n-k}\right) \int_{0}^{t} g(s) I_{k}\left(h^{\otimes k} \mathbf{1}_{[0, s)}^{\otimes k}\right) d \tilde{N}_{s} .
\end{aligned}
$$

By linearity, for $n \geq 1$, the statement is now completely proved for every finite linear combination of processes with the form (22), and a standard density argument yields the result for every process with the form

$$
v_{s}=I_{n}\left(g(\cdot, s) \mathbf{1}_{[0, s) \cup(t, 1]}^{\otimes n}\right), \quad s \in T,
$$

where the function $g\left(x_{1}, \ldots, x_{n}, s\right)$ is an element of $L^{2}\left(T^{n+1}\right)$ and is symmetric in the variables $\left(x_{1}, \ldots, x_{n}\right)$. To deal with the general case, suppose that $u_{s}=\sum_{n \geq 0} I_{n}\left(h_{n}(\cdot, s)\right) \in L^{2}(T \times \Omega)$ is $\mathbb{F}_{(s, t]^{c}}$ - predictable on $[0, t]$. This implies, in particular, by setting $h_{n}^{t}\left(s, t_{1}, \ldots, t_{n}\right)=\mathbf{1}_{[0, t]}(s)$ $h_{n}\left(t_{1}, \ldots, t_{n}, s\right)$,

$$
\begin{aligned}
\sum_{n=0}^{\infty} n ! \int_{0}^{t} d s\left\|h_{n}(\cdot, s)\right\|_{n}^{2} & =\sum_{n=0}^{\infty} n ! \int_{0}^{1} d s\left\|h_{n}^{t}(\cdot, s)\right\|_{n}^{2}=\sum_{n=0}^{\infty} n !\left\|h_{n}^{t}\right\|_{n+1}^{2}<+\infty, \text { and } \\
I_{n}\left(h_{n}(\cdot, s)\right) & =I_{n}\left(h_{n}(\cdot, s) \mathbf{1}_{[0, s) \cup(t, 1]}^{\otimes n}\right), n \geq 1, s \in[0, t] .
\end{aligned}
$$

Now observe that, thanks to the previous discussion,

$$
(n+1) !\left\|\widetilde{h_{n}^{t}}\right\|_{n+1}^{2}=\mathbf{E} I_{n+1}\left(\widetilde{h_{n}^{t}}\right)^{2}=n ! \int_{0}^{t}\left\|h_{n}(\cdot, s)\right\|_{n}^{2} d s
$$

implying

$$
\sum_{n=0}^{\infty}(n+1) !\left\|\widetilde{h_{n}^{t}}\right\|_{n+1}^{2}=\sum_{n=0}^{\infty} n !\left\|h_{n}^{t}\right\|_{n+1}^{2}<+\infty,
$$

and therefore $u \mathbf{1}_{[0, t]} \in \operatorname{Dom}(\delta)$. The conclusion is achieved by standard arguments. 
(ii) Thanks to [10, Theorem 4.5], we obtain immediately, for $s \leq t$,

$$
G=\mathbf{E}\left(G \mid \mathbb{F}_{s}\right)+\int_{s}^{t}{ }^{(p)}\left(D_{r} G\right) d \tilde{N}_{r}
$$

where $^{(p)}(\cdot)$ indicates the predictable projection operator with respect to $\mathbb{F}_{s}, s \leq t$. To conclude, it is sufficient to use the independence of the increments of $\tilde{N}$ to obtain that, for every $s \leq r \leq t$,

$$
\begin{aligned}
\mathbf{E}\left(G \mid \mathbb{F}_{s}\right) & =\mathbf{E}\left(G \mid \mathbb{F}_{(s, t]^{c}}\right) \\
{ }^{(p)}\left(D_{r} G\right) & =\mathbf{E}\left(D_{r} G \mid \mathbb{F}_{r-}\right)=\mathbf{E}\left(D_{r} G \mid \mathbb{F}_{[r, t]^{c}}\right)={ }^{(p, t)}\left(D_{r} G\right),
\end{aligned}
$$

a.s. $-\mathbf{P}$.

Remark 2 (i) The arguments used in the proof of Proposition 1-(i) are exclusively based on the covariance structure of multiple integrals and formula (16), and they can be directly applied to the Brownian case. This implies, for instance, that the Skorohod integral appearing in the statement of Proposition A.1 in [13] is also a martingale stochastic integral with respect to an independently enlarged Brownian filtration.

(ii) We stress that, for the moment, we require the functional $G$, in part (ii) of Proposition 1 , to be $\mathbb{F}_{t}$ - measurable. In the next section we will show that (21) holds indeed for every $G \in \mathbb{D}^{1,2}$. For $t=1$, Proposition 1-(ii) has also been proved in [1].

Define $\mathbb{S}^{*}$ to be the (dense) subset of $L^{2}(T \times \Omega)$ and $\mathbb{L}^{k, 2}, k \geq 1$, composed of processes of the type

$$
v_{s}=\sum_{n=0}^{N} I_{n}\left(f_{n}(s, \cdot)\right), \quad s \in T
$$

where $N<+\infty$, and, for every $n, f_{n}\left(s, t_{1}, \ldots, t_{n}\right) \in S_{n+1}$ and $f_{n}$ is symmetric in the variables $\left(t_{1}, \ldots, t_{n}\right)$. Then, for every $v \in \mathbb{S}^{*}$, a classic characterization of predictable projections (see 5 . Theorem 6.43]) implies immediately, thanks to formulae (4) and (16), that there exists a jointly measurable application $\phi_{v}$

$$
\Omega \times \Delta^{2} \mapsto \mathbb{R}:(\omega ; s, t) \mapsto \phi_{v}(\omega ; s, t)
$$

where $\Delta^{2}=\left\{(s, t) \in[0,1]^{2}: 0 \leq s \leq t\right\}$, such that, for every $(s, t) \in \Delta^{2}, \phi_{v}(\cdot ; s, t)$ is a version of $\mathbf{E}\left[v_{s} \mid \mathbb{F}_{\left.[s, t]^{c}\right]}\right.$ and, a.s. - $\mathbf{P}$,

$$
\phi_{v}(\cdot ; s, t)={ }^{(p, t)}\left(v_{s}\right)
$$

In general, due again to [5] Theorem 6.43], for every fixed $t \in(0,1]$ and every process $u \in L^{2}(T \times \Omega)$, the associated predictable projection $(p, t)\left(u_{s}\right)$ is such that, for every $s \in[0, t], \mathbf{E}\left[u_{s} \mid \mathbb{F}_{[s, t]^{c}}\right]=$

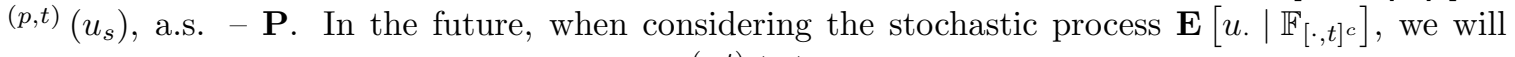
implicitly refer to its predictable modification ${ }^{(p, t)}(u$.). For instance, with such a convention, formula (21) can be unambiguously rewritten as

$$
G=\mathbf{E}\left(G \mid \mathbb{F}_{(s, t]^{c}}\right)+\int_{s}^{t} \mathbf{E}\left[u_{r} \mid \mathbb{F}_{[r, t]^{c}}\right] d \tilde{N}_{r} .
$$




\section{Forward-backward martingales and approximation of an- ticipating integrals}

In this section, we explore the connection between the anticipating integrals of the form (ID), and a special class of usual Itô integrals. This relation is applied to prove that, just as on the Wiener space, anticipating integral processes can be represented as the limit, under a certain norm, of linear combinations of products of forward and backward martingales. We start by adapting to the Poisson situation some known results on the Wiener space. In particular, we will need the following generalized Clark-Ocone formula, which extends Proposition 1 above and is the actual equivalent, on the Poisson space, of [13, Proposition A.1].

Proposition 2 Let the notation of the previous section prevail, and let $G \in \mathbb{D}^{1,2}$. Then, for every $0 \leq s \leq t \leq 1$, formula [21) holds. We have also the relation, for $s>0$,

$$
G=\mathbf{E}\left(G \mid \mathbb{F}_{[s, t]^{c}}\right)+\int_{s-}^{t}{ }^{(p, t)}\left(D_{r} G\right) d \tilde{N}_{r}=\mathbf{E}\left(G \mid \mathbb{F}_{[s, t]^{c}}\right)+\delta\left({ }^{(p, t)}(D . G) \mathbf{1}_{[s, t]}(\cdot)\right)
$$

where $\int_{s-}^{t}{ }^{(p, t)}\left(D_{r} G\right) d \tilde{N}_{r}=\lim _{\alpha \uparrow s} \int_{\alpha}^{t}{ }^{(p, t)}\left(D_{r} G\right) d \tilde{N}_{r}$.

Proof. First observe that the second equality in $(23)$ follows from the $\mathbb{F}_{(\cdot, t]^{c}}$ - predictability of ${ }^{(p, t)}(D . G)$, and an application of Proposition 1-(i). Moreover, thanks to the martingale property of stochastic integrals, it is sufficient to prove the statement for $s=0$ and $t \in(0,1]$. We start by considering a random variable $G \in \mathbb{D}^{1,2}$ of the form

$$
G=I_{m}\left(h \mathbf{1}_{[0, t]}^{\otimes m}\right) \times I_{n}\left(g \mathbf{1}_{(t, 1]}^{\otimes n}\right):=G_{1} \times G_{2}, \quad n, m \geq 0,
$$

where, for $n, m \geq 1, h \in L_{s}^{2}\left([0,1]^{m}\right), g \in L_{s}^{2}\left([0,1]^{n}\right)$, and $I_{0}$ stands for a real constant. Random variables such as (24) are total in $\mathbb{D}^{1,2}$. Moreover, we can apply Proposition 1 to $G_{1}$ and obtain, thanks to the stochastic independence between $\mathbb{F}_{t^{c}}$ and $\mathbb{F}_{t}$ and by (15)

$$
\begin{aligned}
G & =G_{2} \times\left[\mathbf{E}\left(G_{1} \mid \mathbb{F}_{t^{c}}\right)+\int_{0}^{t}{ }^{(p, t)}\left(D_{r} G_{1}\right) d \tilde{N}_{r}\right] \\
& =\mathbf{E}\left(G \mid \mathbb{F}_{t^{c}}\right)+\int_{0}^{t} G_{2} \times{ }^{(p, t)}\left(D_{r} G_{1}\right) d \tilde{N}_{r} \\
& =\mathbf{E}\left(G \mid \mathbb{F}_{t^{c}}\right)+\int_{0}^{t}{ }^{(p, t)}\left(G_{2} \times D_{r} G_{1}\right) d \tilde{N}_{r} .
\end{aligned}
$$

Note that the last equality comes from [5] Corollary 6.44] and from the fact that $G_{2}$ is $\mathbb{F}_{t^{c}}$ measurable, implying that the (constant) application $r \mapsto G_{2}, r \in[0, t]$, can be interpreted as a $\mathbb{F}_{(r, t]^{c}}$ - predictable process. Finally, we observe that (9) and (16) imply that

$$
G_{2} \times D_{r} G_{1}=D_{r} G, \text { for every } r \in[0, t],
$$

so that, by linearity, (21) is completely proved for every finite linear combination of random variables such as (24). Now suppose that a certain sequence $G^{(n)} \in \mathbb{D}^{1,2}$ enjoys property (21) and that $G^{(n)}$ converges to $G$ in $\mathbb{D}^{1,2}$ as $n$ goes to $+\infty$. Then, $\mathbf{E}\left(G^{(n)} \mid \mathbb{F}_{t^{c}}\right) \rightarrow \mathbb{E}\left(G \mid \mathbb{F}_{t^{c}}\right)$ in $L^{2}(\mathbf{P})$, and moreover the relation

$$
{ }^{(p, t)}\left(D_{r} H\right)=\mathbf{E}\left[D_{r} H \mid \mathbb{F}_{[r, t]^{c}}\right],
$$


true for every fixed $r \in[0, t]$ and every $H \in \mathbb{D}^{1,2}$, implies immediately, thanks to Jensen inequality and the isometric properties of Itô integrals,

$$
\begin{aligned}
\mathbf{E}\left\{\left[\int_{0}^{t}\left[{ }^{(p, t)}\left(D_{r} G^{(n)}\right)-{ }^{(p, t)}\left(D_{r} G\right)\right] d \tilde{N}_{r}\right]^{2}\right\} & =\int_{0}^{t} \mathbf{E}\left[\left[(p, t)\left(D_{r}\left(G^{(n)}-G\right)\right)\right]^{2}\right] d r \\
& \leq \mathbf{E} \int_{0}^{t}\left(D_{r}\left(G^{(n)}-G\right)\right)^{2} d r \rightarrow 0,
\end{aligned}
$$

and therefore

$$
G=\mathbf{E}\left(G \mid \mathbb{F}_{t^{c}}\right)+\int_{0}^{t}{ }^{(p, t)}\left(D_{r} G\right) d \tilde{N}_{r}
$$

To obtain (21), use the totality in $\mathbb{D}^{1,2}$ of random variables such as (24). Eventually, to prove (23) just write, for $\varepsilon>0$,

$$
G=\mathbf{E}\left(G \mid \mathbb{F}_{(s-\varepsilon, t]^{c}}\right)+\int_{s-\varepsilon}^{t}{ }^{(p, t)}\left(D_{r} G\right) d \tilde{N}_{r}
$$

so that, by letting $\varepsilon \downarrow 0$, the conclusion follows from the fact that the paths of Itô stochastic integrals (with respect to $\tilde{N}$ ) are càdlàg, as well as relation (20) and a standard martingale argument.

Remark 3 Proposition 2 can also be proved along the same lines of the proof of [13, Proposition A.1]. Suppose indeed that $G$ admits the chaotic decomposition

$$
G=\sum_{m \geq 0} I_{m}\left(g_{m}\right), \quad g_{m} \in L_{s}^{2}\left(T^{m}\right) .
$$

Then, by (8) and (16) $), D_{r} G=\sum_{m \geq 1} m I_{m-1}\left(g_{m}(\cdot, r)\right)$ and

$$
\mathbf{E}\left(D_{r} G \mid \mathbb{F}_{[r, t]^{c}}\right)=\sum_{m \geq 1} m I_{m-1}\left(g_{m}(\cdot, r) \mathbf{1}_{[r, t]^{c}}^{\otimes(m-1)}\right) .
$$

Since the symmetrization in the $m$ variables $r, t_{1}, \ldots, t_{m-1}$ of the function $\mathbf{1}_{[s, t]}(r) \mathbf{1}_{[r, t]^{c}}^{\otimes(m-1)}\left(t_{1}, \ldots, t_{m-1}\right)$ is given by $\frac{1}{m} \mathbf{1}_{A_{m}}$ where $A_{m}=\bigcup_{i=1}^{m}\left\{\left(t_{1}, \ldots, t_{m}\right) \in T^{m}, t_{i} \in[s, t]\right\}$ we get

$$
\begin{aligned}
\delta\left(\mathbf{1}_{[s, t]}(\cdot) \mathbf{E}\left(D . G \mid \mathbb{F}_{[\cdot, t]^{c}}\right)\right) & =\sum_{m \geq 1} I_{m}\left(g_{m} \mathbf{1}_{A_{m}}\right) \\
& =\sum_{m \geq 0} I_{m}\left(g_{m}\right)-\sum_{m \geq 0} I_{m}\left(g_{m} \mathbf{1}_{A_{m}^{c}}\right) \\
& =G-\mathbf{E}\left(G \mid \mathbb{F}_{[s, t]^{c}}\right) .
\end{aligned}
$$

The discussion of Paragraph 2.2 can be used to interpret the Skorohod integral on the left side as an Itô integral of a predictable process.

The next Proposition shows that every indefinite anticipative integral $\int_{0}^{t} u_{s} \delta \tilde{N}_{s}$ can be written, at fixed $t \in[0,1]$, as an Itô-Skorohod integral with the form $\int_{0}^{t} \mathbf{E}\left(w_{s} \mid \mathbb{F}_{[s, t]^{c}}\right) d \tilde{N}_{s}$ where $w$ can be explicitly given in terms of $u$. 
Proposition 3 Let $X$ be a Skorohod integral process $X_{t}=\delta\left(u 1_{[0, t]}\right), t \in[0,1]$, with $u \in \mathbb{L}^{k, 2}, k \geq 3$. Then, there exists a unique process $w \in \mathbb{L}^{k-2,2}$, independent of $t$, such that, for every fixed $t$,

$$
X_{t}=\int_{0}^{t} \mathbf{E}\left(w_{s} \mid \mathbb{F}_{[s, t]^{c}}\right) d \tilde{N}_{s}=\delta\left(\mathbf{E}\left(w . \mid \mathbb{F}_{[\cdot, t]^{c}}\right) \mathbf{1}_{[0, t]}(\cdot)\right) \text {, a.s. }-\mathbf{P} .
$$

Proof. By applying the Clark-Ocone type formula (23) to the integrand $u$ we can write

$$
\begin{aligned}
X_{t} & =\int_{0}^{t} u_{s} d \tilde{N}_{s} \\
& =\int_{0}^{t} \mathbf{E}\left(u_{s} \mid \mathbb{F}_{[s, t]^{c}}\right) d \tilde{N}_{s}+\int_{0}^{t}\left(\int_{s-}^{t} \mathbf{E}\left(D_{r} u_{s} \mid \mathbb{F}_{[r, t]^{c}}\right) \delta \tilde{N}_{r}\right) \delta \tilde{N}_{s} .
\end{aligned}
$$

Using a Fubini type theorem (that we can argue exactly as in [15], by using working on the chaotic expansions) we can interchange the two Skorohod integrals appearing in the second term to obtain that

$$
\begin{aligned}
X_{t} & =\int_{0}^{t} \mathbf{E}\left(u_{s} \mid \mathbb{F}_{[s, t]^{c}}\right) d \tilde{N}_{s}+\int_{0}^{t}\left(\int_{0}^{r} \mathbf{E}\left(D_{r} u_{s} \mid \mathbb{F}_{[r, t]^{c}}\right) \delta \tilde{N}_{s}\right) \delta \tilde{N}_{r} \\
& =\int_{0}^{t} \mathbf{E}\left(u_{s} \mid \mathbb{F}_{[s, t]^{c}}\right) d \tilde{N}_{s}+\int_{0}^{t} \mathbf{E}\left(\int_{0}^{r} D_{r} u_{s} \delta \tilde{N}_{s} \mid \mathbb{F}_{[r, t]^{c}}\right) \delta \tilde{N}_{r} \\
& =\int_{0}^{t} \mathbf{E}\left(w_{s} \mid \mathbb{F}_{[s, t]^{c}}\right) d \tilde{N}_{s}
\end{aligned}
$$

where we used Proposition 1-(i) as well as the fact that the increments of the Poisson process are independent and we adopted the notation

$$
w_{s}:=u_{s}+\delta\left(D_{s} u . \mathbf{1}_{[0, s]}(\cdot)\right):=u_{s}+v_{s} .
$$

Let us show that the process $w$ introduced in (26) belongs to $\mathbb{L}^{k-2,2}$. It suffices indeed to prove that $v \in \mathbb{L}^{k-2,2}$. Thanks to the inequality (11) and formula (14), we can write

$$
\begin{aligned}
\|v\|_{1,2}^{2}= & \mathbf{E} \int_{0}^{1} \delta\left(D_{s} u \mathbf{1}_{[0, s]}\right)^{2} d s+\mathbf{E} \int_{0}^{1} \int_{0}^{1}\left(D_{\alpha} \delta\left(D_{s} u \mathbf{1}_{[0, s]}\right)\right)^{2} d s d \alpha \\
\leq & 2 \mathbf{E} \int_{0}^{1} \int_{0}^{1}\left(D_{r} u_{s}\right)^{2} d r d s+\mathbf{E} \int_{0}^{1} \int_{0}^{1} \int_{0}^{1}\left(D_{\alpha} D_{r} u_{s}\right)^{2} d s d r d \alpha \\
& +\mathbf{E} \int_{0}^{1} \int_{0}^{1} \delta\left(D_{\alpha} D_{s} u \mathbf{1}_{[0, s]}\right)^{2} d s d \alpha \\
\leq & 2 \mathbf{E} \int_{0}^{1} \int_{0}^{1}\left(D_{r} u_{s}\right)^{2} d r d s+2 \mathbf{E} \int_{0}^{1} \int_{0}^{1} \int_{0}^{1}\left(D_{\alpha} D_{r} u_{s}\right)^{2} d s d r d \alpha \\
& +\mathbf{E} \int_{0}^{1} \int_{0}^{1} \int_{0}^{1} \int_{0}^{1}\left(D_{\beta} D_{\alpha} D_{s} u_{r}\right)^{2} d r d s d \alpha d \beta<+\infty,
\end{aligned}
$$

since $u \in \mathbb{L}^{3,2}$. In general, it can be similarly proved that

$$
\|v\|_{k-2,2}^{2} \leq C_{k}\|v\|_{k, 2}^{2}, \quad k \geq 3,
$$


where $C_{k}$ is a positive constant depending exclusively on $k$. Concerning the uniqueness, let us suppose that there exists another process $w^{\prime} \in \mathbb{L}^{k-2,2}$ such that $X_{t}=\int_{0}^{t} \mathbf{E}\left(w_{s}^{\prime} \mid \mathbb{F}_{[s, t]^{c}}\right) d \tilde{N}_{s}$. Then, if $z_{s}=w_{s}-w_{s}^{\prime}$, we get

$$
\int_{0}^{t} \mathbf{E}\left(z_{s} \mid \mathbb{F}_{[s, t]^{c}}\right) d \tilde{N}_{s}=0
$$

and therefore, for every $t \in(0,1]$,

$$
\int_{0}^{t} \mathbf{E}\left[\mathbf{E}\left(z_{s} \mid \mathbb{F}_{[s, t]^{c}}\right)\right]^{2} d s=0 .
$$

Let us assume that $z$ has the chaotic expression $z_{s}=\sum_{m \geq 0} I_{m}\left(g_{m}(\cdot, s)\right)$ where $g_{m} \in L^{2}\left(T^{m+1}\right)$; note that, for $m \geq 2$, the function $g_{m}\left(s_{1}, \ldots, s_{m}, s\right)$ can be taken to be symmetric in the variables $\left(s_{1}, \ldots, s_{m}\right)$. The above condition ensures that

$$
\sum_{m \geq 0} \int_{0}^{t}\left(\int_{[s, t]^{c}} \ldots \int_{[s, t]^{c}} g_{m}^{2}\left(s_{1}, \ldots, s_{m}, s\right) d s_{1} \ldots d s_{m}\right) d s=0
$$

and thus

$$
\int_{[s, t]^{c}} \ldots \int_{[s, t]^{c}} g_{m}^{2}\left(s_{1}, \ldots, s_{m}, s\right) d s_{1} \ldots d s_{m}=0
$$

for every $m \geq 0$ and for almost every $s \in[0, t]$. By letting $s \rightarrow t$ we get that $g_{m}(\cdot)=0$ almost everywhere on $T^{m+1}$ and the conclusion is obtained.

Remark 4 (i) We claim that it is possible to prove a converse to Proposition 3. More precisely, as in [28], we can show that if, for a fixed $t, Y_{t}=\int_{0}^{t} \mathbf{E}\left(w_{s} \mid \mathbb{F}_{[s, t]^{c}}\right) d \tilde{N}_{s}$ with $w \in \mathbb{L}^{1,2}$, then there exists $u \in \operatorname{Dom}(\delta)$ such that $Y_{t}=\int_{0}^{t} u_{s} \delta \tilde{N}_{s}$. The proof would use an analogue of the characterization of Skorohod integrals stated in Proposition 2.1. of [11] for the Wiener case; one can prove a similar characterization in the Poisson context, by following the same line of reasoning as in [11, Proposition 2.1]. This point will be discussed in a separate paper.

(ii) A question that is not likely to be answered as easily as in the Wiener case (see [28]), is when a Skorohod integral $X_{t}$ as in (1) and an Itô-Skorohod integral such as $Y_{t}=\int_{0}^{t} \mathbf{E}\left(w_{s} \mid \mathbb{F}_{[s, t] c}\right) d \tilde{N}_{s}$ are indistinguishable as stochastic processes, and not only reciprocal modifications. This is not immediate to answer since, on the Poisson space, we do not know any sufficiently general criterion, ensuring that an anticipating integral admits a (right)-continuous version. Nevertheless, as shown in the following example, one can sometimes apply classical results from the general theory of stochastic processes.

Example (Indefinite integrals that are indistinguishable from Itô-Skorohod processes). Let the process $X_{t}, t \in[0,1]$, be defined as in (11), and assume that the integrand $u$ and all its Malliavin derivatives $D^{k} u$ are bounded by a deterministic constant, uniformly on $T^{k} \times \Omega$. Then, the assumptions of Proposition 3 are verified, and we immediately deduce the existence of an Itô -Skorohod integral with the form $Y_{t}=\int_{0}^{t} \mathbf{E}\left(w_{s} \mid \mathbb{F}_{[s, t]^{c}}\right) d \tilde{N}_{s}, t \in[0,1]$, such that $X$ and $Y$ are modifications. We claim that, in this setting, $X$ and $Y$ also admit two indistinguishable càdlàg modifications. Recall indeed the following classic criterion (see [6, Ch. III, Section 4]): if $Z$ is a stochastic process such that for some $p \geq 1$ and $\beta>0$,

$$
\mathbf{E}\left|\left(Z_{t+h}-Z_{t}\right)\left(Z_{t}-Z_{t-h}\right)\right|^{p} \leq C(p) \times h^{1+\beta},
$$


where $C(p)$ is a positive constant, then $Z$ admits a càdlàg modification. Note that if $Z^{\prime}$ is a modification of $Z$, and $Z$ satisfies (27), then (27) must also hold for $Z^{\prime}$. We shall prove (27) for $Z=X$. To this end, note $I_{h+}=[t, t+h]$ and $I_{h-}=[t-h, t]$, so that, under the above assumptions and by (12),

$$
\begin{aligned}
& \mathbf{E}\left|\left(X_{t+h}-X_{t}\right)\left(X_{t}-X_{t-h}\right)\right|^{2}=\mathbf{E}\left(\delta\left(u \mathbf{1}_{I_{h+}}\right) \delta\left(u \mathbf{1}_{I_{h+}}\right) \delta\left(u \mathbf{1}_{I_{h-}}\right)^{2}\right) \\
& =\mathbf{E} \int_{I_{h+}} u_{s} D_{s}\left[\delta\left(u \mathbf{1}_{I_{h+}}\right) \delta\left(u \mathbf{1}_{I_{h-}}\right)^{2}\right] d s=\mathbf{E}\left(\int_{I_{h+}} u_{s}\left[D_{s} \delta\left(u \mathbf{1}_{I_{h+}}\right)\right] d s\right) \delta\left(u \mathbf{1}_{I_{h-}}\right)^{2} \\
& +\mathbf{E}\left(\int_{I_{h+}} u_{s}\left[D_{s} \delta\left(u \mathbf{1}_{I_{h-}}\right)^{2}\right] d s\right) \delta\left(u \mathbf{1}_{I_{h+}}\right)+\mathbf{E}\left(\int_{I_{h+}} u_{s}\left[D_{s} \delta\left(u \mathbf{1}_{I_{h+}}\right)\right]\left[D_{s} \delta\left(u \mathbf{1}_{I_{h-}}\right)^{2}\right] d s\right) \\
& :=A+B+C .
\end{aligned}
$$

By formula (14), the first summand above can be decomposed as follows

$$
A=\mathbf{E} \int_{I_{h+}} u_{s}\left[u_{s} \mathbf{1}_{h+}(s)+\delta\left(D_{s} u \mathbf{1}_{h+}\right)\right] d s:=A_{1}+A_{2} .
$$

We need only show how to handle $A_{1}$, and similar techniques can be used to deal with the remaining terms $A_{2}, B$ and $C$. Indeed, we can write

$$
A_{1}=\mathbf{E} \delta\left(u \mathbf{1}_{I_{h-}}\right)^{2} \int_{I_{h+}} u_{s}^{2} d s \leq c s t \times h \mathbf{E} \delta\left(u \mathbf{1}_{I_{h-}}\right)^{2} \leq c s t \times h^{2}
$$

since, by (11),

$$
\mathbf{E} \delta\left(u \mathbf{1}_{I_{h-}}\right)^{2} \leq \mathbf{E} \int_{I_{h-}} u_{s}^{2} d s+\int_{I_{h-}} \int_{I_{h-}}\left(D_{s} u_{\alpha}\right)^{2} d s d \alpha \leq c s t \times h
$$

because $u$ and its derivative are assumed to be uniformly bounded. Since (27) is also true for $Z=Y$, we deduce that there exist two processes $X^{\prime}$ and $Y^{\prime}$ such that $X^{\prime}$ is a càdlàg modification of $X$ and $Y^{\prime}$ is a càdlàg modification of $Y$. We can now apply a classic argument (see for instance [23. Theorem 2, p. 4]), to deduce that $X^{\prime}$ and $Y^{\prime}$ are also indistinguishable.

Let us recall some notation taken from $\left[18\right.$. By $L_{0}^{2}(\mathbf{P})$ we denote the set of zero mean square integrable random variables. We will write $\mathbf{B F}$ for the class of stochastic processes that can be expressed as finite linear combinations of processes of the type

$$
Z_{t}=\mathbf{E}\left(H_{1} \mid \mathbb{F}_{t}\right) \times \mathbf{E}\left(H_{2} \mid \mathbb{F}_{t^{c}}\right)=M_{t} \times M_{t}^{\prime}
$$

where $H_{1} \in L_{0}^{2}(\mathbf{P})$ and $H_{2} \in L^{2}(\mathbf{P})$. Plainly, $M$ is a martingale with respect to $\mathbb{F}_{t}$ and $M^{\prime}$ is a backward martingale. By backward martingale we mean that $M_{t}^{\prime}$ is in $L^{1}(\mathbf{P})$ and $\mathbb{F}_{t^{c}}$ - measurable for every $t$, and $\mathbf{E}\left(M_{s}^{\prime} \mid \mathbb{F}_{t^{c}}\right)=M_{t}^{\prime}$ for any $s \leq t$; see e.g. 24].

We give a counterpart of Lemma 2 in [18]. The proof needs a slightly different argument.

Lemma 1 Let $A_{1}, A_{2}$ be two disjoint Borel subsets of $[0,1]$ and assume that $F$ is a random variable in $\mathbb{D}^{k, 2}, k \geq 1$, such that $F$ is measurable with respect to the $\sigma$-algebra $\mathbb{F}_{A_{1}} \vee \mathbb{F}_{A_{2}}$. Then, $F$ is the limit in $\mathbb{D}^{k, 2}$ of linear combinations of smooth random variables of the type

$$
G=G_{1} \times G_{2},
$$

where, for $i=1,2, G_{i}$ is a polynomial, $\mathbb{F}_{A_{i}}$ - measurable functional. 
Proof. Suppose first that $F$ is a simple functional of the form

$$
F=\tilde{N}\left(h_{1}\right) \ldots \tilde{N}\left(h_{n}\right)
$$

where $n \geq 1$ and $h_{i} \in L^{2}(T), i=1, \ldots, n$. Then, the conclusion can be obtained exactly as in Lemma 2 of [18, by using twice formula (16). The next step is to consider $F=I_{n}(f)$ with $f \in L^{2}\left(T^{n}\right)$. In this case, by the above discussed definition of the multiple integral $I_{n}, F$ is the limit in $\mathbb{D}^{k, 2}$ of random variables $F_{k}$ as in (29). Let us denote by $p_{m, k}$ a sequence of linear combinations of product (28) such that $p_{m, k} \rightarrow F_{k}$ in $\mathbb{D}^{k, 2}$ as $m \rightarrow \infty$. Clearly,

$$
\left\|F-p_{m, k}\right\|_{k, 2} \leq\left\|F-F_{k}\right\|_{k, 2}+\left\|F_{k}-p_{m, k}\right\|_{k, 2}
$$

and this goes to zero when $m, k \rightarrow \infty$. Eventually, take the general case $F=\sum_{m>0} I_{m}\left(f_{m}\right)$ where $f_{m} \in L^{2}\left(T^{n}\right)$ are symmetric functions. The conclusion will follow if we prove that $F$ can be approximated in $\mathbb{D}^{k, 2}$ by a sequence $F^{N}$ of random variables with finite chaotic expansion and this is trivial if we put $F^{N}=\sum_{n=0, \ldots, N} I_{n}\left(f_{n}\right)$. See also Proposition 1.2.1 in [12] for further details.

Remark 5 Recall the relation between multiple integrals and Charlier polynomials stated in formula (6). Then, by inspection of the proof of Lemma 1 (which partially follows that of Lemma 2 in [18] ), and thanks to the multiplication formula (7), it is clear that if $F=I_{n}(f)\left(\in \mathbb{D}^{k, 2}\right)$, then $F$ can be approximated in $\mathbb{D}^{k, 2}$ by linear combinations of random variables with the form $C_{n}\left(t_{k+1}-t_{k}, \tilde{N}_{t_{k+1}}-\tilde{N}_{t_{k}}\right)$ where $0 \leq t_{k}<t_{k+1} \leq 1$, and $C_{n}$ is the nth Charlier polynomial. Therefore, the random variables $G_{i}, i=1,2$, appearing in the proof of Lemma 1 can be chosen as polynomial functionals of degrees $d_{i}, i=1,2$, such that $d_{1}+d_{2} \leq n$.

We shall also introduce the following quadratic variation (in mean) of a given measurable process $\left\{X_{t}: t \in T\right\}$ such that $\mathbf{E} X_{t}^{2}<+\infty$ for every $t$ :

$$
V(X)=\sup _{\pi} \mathbf{E} \sum_{i=0}^{n-1}\left(X_{t_{i+1}}-X_{t_{i}}\right)^{2}
$$

where $\pi$ runs over all partitions of $T=[0,1]$, with the form $0=t_{0}<t_{1}<\ldots<t_{n}=1$.

We state the main result of this section.

Theorem 1 Let $X$ be a Skorohod integral process $X_{t}=\delta\left(u \mathbf{1}_{[0, t]}\right)$ with $u \in \mathbb{L}^{k, 2}, k \geq 3$. Then there exists a sequence of processes $\left(Z_{t}^{(r)}\right)_{t \in[0,1]}, r \geq 1$ such that

$$
Z^{(r)} \in \mathbf{B F} \text { for every } r \geq 1
$$

and

$$
\lim _{r \rightarrow \infty} V\left(X-Z^{(r)}\right)=0 .
$$

Proof. Remark first that $V(X)<\infty$ by Proposition 1 in [27]. We will use the ItôSkorohod representation of $X_{t}=\int_{0}^{t} \mathbf{E}\left(w_{s} \mid \mathbb{F}_{[s, t]^{c}}\right) d \tilde{N}_{s}$ with $w$ given by (26). For $n \geq 1$ and a partition $\pi=\left\{0=t_{0}<\ldots<t_{n}=1\right\}$, we introduce the approximation of $w$

$$
w_{t}^{\pi}=\sum_{i=0}^{n-1} \frac{1}{t_{i+1}-t_{i}}\left(\int_{t_{i}}^{t_{i+1}} \mathbf{E}\left(w_{s} \mid \mathbb{F}_{\left[t_{i}, t_{i+1}\right]^{c}}\right) d s\right) 1_{\left(t_{i}, t_{i+1}\right]}(t):=\sum_{i=0}^{n-1} F_{i} \mathbf{1}_{\left(t_{i}, t_{i+1}\right]}(t), \quad t \in[0,1] .
$$


Since $w \in \mathbb{L}^{1,2}$ then $w^{\pi} \in \mathbb{L}^{1,2}$ and $w^{\pi}$ converges to $w$ in $\mathbb{L}^{1,2}$ as $|\pi| \rightarrow 0$ (see [27, and also [12] for the Gaussian case). Note that the random variables $F_{i}, i=0, \ldots, n$, appearing in (32) are measurable

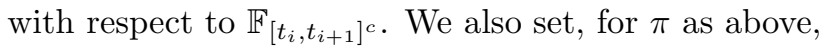

$$
Y_{t}^{\pi}=\delta\left(\mathbf{E}\left(w_{.}^{\pi} \mid \mathbb{F}_{[\cdot, t]^{c}}\right) \mathbf{1}_{[0, t]}\right)=\int_{0}^{t} \mathbf{E}\left(w_{s}^{\pi} \mid \mathbb{F}_{[s, t]^{c}}\right) d \tilde{N}_{s}, \quad t \in T .
$$

Using properties (16), (17) and (15) of Poisson Skorohod integrals we therefore deduce

$$
\begin{aligned}
Y_{t}^{\pi} & =\sum_{i=0}^{n-1} \int_{0}^{t} 1_{\left(t_{i}, t_{i+1}\right]}(s) \mathbf{E}\left(F_{i} \mid \mathbb{F}_{[s, t]^{c}}\right) \delta \tilde{N}_{s} \\
& =\sum_{i=0}^{n-1} \int_{0}^{t} 1_{\left(t_{i}, t_{i+1}\right]}(s) \mathbf{E}\left(F_{i} \mid \mathbb{F}_{\left[t_{i}, t_{i+1} \vee t\right]^{c}}\right) \delta \tilde{N}_{s} \\
& =\sum_{i=0}^{n-1} \mathbf{E}\left(F_{i} \mid \mathbb{F}_{\left(t_{i}, t_{i+1} \vee t\right]^{c}}\right)\left(\tilde{N}_{t \wedge t_{i+1}}-\tilde{N}_{t_{i}}\right) \mathbf{1}_{\left(t \geq t_{i}\right)}
\end{aligned}
$$

note, in particular, that the last equality in (33) derives from an application of formula (15), where the last two terms vanish thanks to (17) (alternatively, one can also use Proposition 1-(i)). This is all we need to conclude the proof of Theorem 1. As a matter of fact, as in [18, proof of Theorem 1], we can now use Lemma 1 to prove that $Y^{\pi}$ (and hence $X$ ) can be approximated, in the sense of formula (31), by a sequence of processes $Z^{(r)}$ satisfying (30).

We state a converse result to Theorem 1; the arguments of [18. Theorem 4] apply, and the proof is therefore omitted. It shows that the " $V$-norm" is somewhat complete.

Theorem 2 Let $Z^{(n)} \in \mathbf{B F}, n \geq 1$ be such that $V\left(Z^{(n)}\right)<\infty$ and $Z^{(n)}$ is a Cauchy sequence in the $V$-norm, in the sense that

$$
\lim _{n, m \rightarrow \infty} V\left(Z^{(n)}-Z^{(m)}\right)=0 .
$$

Then, there exists a Skorohod integral process $X$ with $V(X)<\infty$ such that

$$
\lim _{n \rightarrow \infty} V\left(Z^{(n)}-X\right)=0
$$

\section{On the stochastic calculus for anticipating integrals on the Poisson space}

In the previous Section we have seen that, for every fixed $t \in[0,1]$, the Skorohod integral $X_{t}=$ $\int_{0}^{t} u_{s} \delta \tilde{N}_{s}$ is equal to the Itô-Skorohod integral $Y_{t}=\int_{0}^{t} \mathbf{E}\left(w_{s} \mid \mathbb{F}_{[s, t]^{c}}\right) d \tilde{N}_{s}$. As seen in Section 2 , the last random variable is the Itô integral of the predictable process $\mathbf{E}\left(w . \mid \mathbb{F}_{[\cdot, t]^{c}}\right)$, with respect to the $\mathbb{F}_{(\cdot, t]^{c}}$ - martingale $\tilde{N}$.. As such, it is an isometry, in the sense that

$$
\mathbf{E}\left(\int_{0}^{t} \mathbf{E}\left(w_{s} \mid \mathbb{F}_{[s, t]^{c}}\right) d \tilde{N}_{s}\right)^{2}=\mathbf{E} \int_{0}^{t} \mathbf{E}\left(w_{s} \mid \mathbb{F}_{[s, t]^{c}}\right)^{2} d s
$$


(this can be also derived from formula (131)), and can moreover be approximated by a sequence of martingales. Define indeed

$$
Y_{t}^{\lambda}:=\int_{0}^{\lambda} \mathbf{E}\left(w_{s} \mid \mathbb{F}_{[s, t]^{c}}\right) d \tilde{N}_{s}=\delta\left(\mathbf{1}_{[0, \lambda]}(\cdot) \mathbf{E}\left(w \cdot \mid \mathbb{F}_{[\cdot, t]^{c}}\right)\right) .
$$

Then, for every fixed $t \in[0,1]$, the process $\left\{Y_{t}^{\lambda}: 0 \leq \lambda \leq t\right\}$ is a martingale with respect to the filtration $\mathbb{F}_{(\lambda, t]^{c}}, \lambda \leq t$, and it holds that $Y_{t}^{t}=Y_{t}$, and for any $\lambda<t$,

$$
\begin{aligned}
\mathbf{E}\left|Y_{t}^{\lambda}-Y_{t}\right|^{2} & =\mathbf{E}\left|\delta\left(\mathbf{1}_{(\lambda, t]}(\cdot) \mathbf{E}\left(w \cdot \mid \mathbb{F}_{[\cdot, t]^{c}}\right)\right)\right|^{2} \\
& =\mathbf{E} \int_{\lambda}^{t} \mathbf{E}\left(w_{s} \mid \mathbb{F}_{[s, t]^{c}}\right)^{2} d s
\end{aligned}
$$

which goes to 0 as $\lambda \rightarrow t$ by the dominated convergence theorem. As a consequence, the $Y_{t}^{\lambda}$ converges in $L^{2}(\mathbf{P})$ to $Y_{t}$ as $\lambda \rightarrow t$ and, by a standard martingale convergence theorem (see e.g. Problem 3.20 in $[8]$ ), the convergence holds a.s. - P. This fact allows us to introduce a stochastic calculus of Itô type for the Itô-Skorohod integral $Y_{t}$ (and hence for indefinite Skorohod integrals $X_{t}$ ). The main idea is to use the tools of the stochastic calculus for the martingale $Y_{t}^{\lambda}$ and to let $\lambda \rightarrow t$. We obtain in this way a change of variable formula for the indefinite integral processes; this seems quite interesting since, as far as we know, there is no Itô formula à la Nualart-Pardoux [13] for anticipating integrals in the Poisson case. We also derive a Burkholder-type bound for the $L^{p}$-norm of a Skorohod integral.

Proposition 4 (Itô's formula) Let $f \in C^{2}(\mathbb{R})$, fixt $\in T$, and define $Y_{t}=\delta\left(\mathbf{E}\left(w \cdot \mid \mathbb{F}_{[\cdot, t]^{c}}\right) \mathbf{1}_{[0, t]}(\cdot)\right)$, where $w \in L^{2}(\Omega \times T)$. Then it holds that

$$
\begin{aligned}
f\left(Y_{t}\right)= & f(0)+\int_{0}^{t} f^{\prime}\left(Y_{t}^{s-}\right) \mathbf{E}\left(w_{s} \mid \mathbb{F}_{[s, t]^{c}}\right) d \tilde{N}_{s} \\
& +\frac{1}{2} \int_{0}^{t} f^{\prime \prime}\left(Y_{t}^{s-}\right) \mathbf{E}\left(w_{s} \mid \mathbb{F}_{[s, t]^{c}}\right)^{2} d s \\
& +\sum_{0 \leq s \leq t}\left(f\left(Y_{t}^{s}\right)-f\left(Y_{t}^{s-}\right)-f^{\prime}\left(Y_{t}^{s-}\right)\left(Y_{t}^{s}-Y_{t}^{s-}\right)\right)
\end{aligned}
$$

where $Y_{t}^{s-}=\lim _{\alpha \rightarrow s, \alpha<s} Y_{t}^{\alpha}$. In particular, let $X_{t}=\int_{0}^{t} u_{s} \delta \tilde{N}_{s}, t \in T$, where $u \in \mathbb{L}^{k, 2}, k \geq 3$, and let $w$ be the process appearing in Proposition 3, formula 25); then, for every $t \in T, f\left(X_{t}\right)$ equals the right-hand side of 35).

Proof. Fix $t \in(0,1]$, and consider the process

$$
\tilde{Y}_{t}^{\lambda}= \begin{cases}Y_{t}^{\lambda} & \text { if } \lambda \leq t \\ Y_{t} & \text { if } t<\lambda<+\infty\end{cases}
$$

as well as the family of $\sigma$-fields

$$
\widetilde{\mathbb{F}}_{\lambda}^{t}= \begin{cases}\mathbb{F}_{(\lambda, t]^{c}} & \text { if } \lambda \leq t \\ \mathbb{F}_{1} & \text { if } t<\lambda<+\infty\end{cases}
$$


then, the application $\lambda \rightarrow \widetilde{Y}_{t}^{\lambda}$ defines a square integrable càdlàg martingale $\widetilde{\mathbb{F}}_{\lambda}^{t}$ with respect to the filtration $\widetilde{\mathbb{F}}_{\lambda}^{t}, \lambda \geq 0$. Moreover,

$$
\begin{aligned}
\tilde{Y}_{t}^{\lambda} & =\int_{0}^{\lambda} \mathbf{E}\left(w_{s} \mid \mathbb{F}_{[s, t]^{c}}\right) \mathbf{1}_{(s \leq t)} d \tilde{N}_{s} \text { and } \\
\left\langle\tilde{Y}_{t}^{\cdot}, \tilde{Y}_{t}^{\cdot}\right\rangle_{\lambda} & =\int_{0}^{t \wedge \lambda} \mathbf{E}\left(w_{s} \mid \mathbb{F}_{[s, t]^{c}}\right)^{2} d s .
\end{aligned}
$$

We can therefore apply Itô's formula (see e.g. [23], Theorem 32, p. 71) at $\lambda=t$ to obtain

$$
\begin{aligned}
f\left(\widetilde{Y}_{t}^{t}\right)= & f\left(Y_{t}\right)=f(0)+\int_{0}^{t} f^{\prime}\left(\widetilde{Y}_{t}^{s-}\right) \mathbf{E}\left(w_{s} \mid \mathbb{F}_{[s, t]^{c}}\right) d \tilde{N}_{s} \\
& +\int_{0}^{t} f^{\prime \prime}\left(\widetilde{Y}_{t}^{s-}\right) \mathbf{E}\left(w_{s} \mid \mathbb{F}_{[s, t]^{c}}\right)^{2} d s \\
& +\sum_{0<s \leq t}\left(f\left(\widetilde{Y}_{t}^{s}\right)-f\left(\widetilde{Y}_{t}^{s-}\right)-f^{\prime}\left(\widetilde{Y}_{t}^{s-}\right)\left(\widetilde{Y}_{t}^{s}-\widetilde{Y}_{t}^{s-}\right)\right) . \\
= & f(0)+\int_{0}^{\lambda} f^{\prime}\left(Y_{t}^{s-}\right) \mathbf{E}\left(w_{s} \mid \mathbb{F}_{[s, t]^{c}}\right) d \tilde{N}_{s} \\
& +\frac{1}{2} \int_{0}^{t} f^{\prime \prime}\left(Y_{t}^{s-}\right) \mathbf{E}\left(w_{s} \mid \mathbb{F}_{[s, t]^{c}}\right)^{2} d s \\
& +\sum_{0<s \leq t}\left(f\left(Y_{t}^{s}\right)-f\left(Y_{t}^{s-}\right)-f^{\prime}\left(Y_{t}^{s-}\right)\left(Y_{t}^{s}-Y_{t}^{s-}\right)\right),
\end{aligned}
$$

by the definition of $\widetilde{Y}$.

We now show that Proposition 4 can be applied to write a change of variables formula for stochastic processes that are representable as the product of a martingale and a backward martingale.

Proposition 5 Let $M$ (resp. $M^{\prime}$ ) be a martingale (resp. a backward martingale) with respect to the filtration $\left\{\mathbb{F}_{t}: t \in T\right\}$, and suppose moreover that $M_{1} \in \mathbb{D}^{1,2}, \mathbf{E}\left(M_{0}\right)=0$ and

$$
\mathbf{E}\left(\int\left(a_{s} M_{s}^{\prime}\right)^{2} d s\right)<+\infty
$$

where $a_{r}=\mathbf{E}\left(D_{r} M_{1} \mid \mathbb{F}_{r-}\right)$. Then, for every $f \in C^{2}(\mathbb{R})$ we have

$$
\begin{aligned}
f\left(M_{t} M_{t}^{\prime}\right)= & f(0)+\int_{0}^{t} f^{\prime}\left(M_{s-} M_{t}^{\prime}\right) M_{t}^{\prime} a_{s} d \tilde{N}_{s} \\
& +\frac{1}{2} \int_{0}^{t} f^{\prime \prime}\left(M_{s-} M_{t}^{\prime}\right)\left(M_{t}^{\prime}\right)^{2} a_{s}^{2} d s \\
& +\sum_{0 \leq s \leq t}\left[f\left(M_{s} M_{t}^{\prime}\right)-f\left(M_{s-} M_{t}^{\prime}\right)-f^{\prime}\left(M_{s-} M_{t}^{\prime}\right) M_{t}^{\prime}\left(M_{s}-M_{s-}\right)\right] .
\end{aligned}
$$


Proof. Remark that a product $M_{t} M_{t}^{\prime}$ is a Itô-Skorohod integral. Indeed, by a standard Clark-Ocone formula (see [10, Theorem 4.5])

$$
M_{t}=\mathbf{E}\left(M_{1} \mid \mathbb{F}_{t}\right)=\int_{0}^{t} \mathbf{E}\left(D_{r} M_{1} \mid \mathbb{F}_{r-}\right) d \tilde{N}_{r}
$$

and using (15) and (17), it holds that

$$
\begin{aligned}
M_{t} M_{t}^{\prime} & =M_{t}^{\prime} \int_{0}^{t} a_{s} d \tilde{N}_{s}=\int_{0}^{t} a_{s} M_{t}^{\prime} d \tilde{N}_{s} \\
& =\int_{0}^{t} a_{s} \mathbf{E}\left(M_{s}^{\prime} \mid \mathbb{F}_{[s, t]^{c}}\right) d \tilde{N}_{s}=\int_{0}^{t} \mathbf{E}\left(a_{s} M_{s}^{\prime} \mid \mathbb{F}_{[s, t]^{c}}\right) d \tilde{N}_{s}
\end{aligned}
$$

By applying Proposition 4, we obtain

$$
\begin{aligned}
M_{t} M_{t}^{\prime}= & f(0)+\int_{0}^{t} f^{\prime}\left(Z_{t}^{s-}\right) \mathbf{E}\left(a_{s} M_{s}^{\prime} \mid \mathbb{F}_{[s, t]^{c}}\right) d \tilde{N}_{s} \\
& +\frac{1}{2} \int_{0}^{t} f^{\prime \prime}\left(Z_{t}^{s-}\right) \mathbf{E}\left(a_{s} M_{s}^{\prime} \mid \mathbb{F}_{[s, t]^{c}}\right)^{2} d s \\
& +\sum_{0 \leq s \leq t}\left(f\left(Z_{t}^{s}\right)-f\left(Z_{t}^{s-}\right)-f^{\prime}\left(Y_{t}^{s-}\right)\left(Z_{t}^{s}-Z_{t}^{s-}\right)\right)
\end{aligned}
$$

where $Z_{t}^{\lambda}=\int_{0}^{\lambda} \mathbf{E}\left(a_{s} M_{s}^{\prime} \mid \mathbb{F}_{[s, t]^{c}}\right) d \tilde{N}_{s}$. The conclusion follows, since for every $\lambda \leq t$, we can write

$$
\begin{aligned}
Z_{t}^{\lambda} & =\mathbb{E}\left(\int_{0}^{\lambda} \mathbf{E}\left(a_{s} M_{s}^{\prime} \mid \mathbb{F}_{[s, \lambda]^{c}}\right) d \tilde{N}_{s} \mid \mathbb{F}_{[\lambda, t]^{c}}\right) \\
& =\mathbb{E}\left(M_{\lambda} M_{\lambda}^{\prime} \mid \mathbb{F}_{[\lambda, t]^{c}}\right)=M_{\lambda-} M_{t}^{\prime}
\end{aligned}
$$

and

$$
Z_{t}^{s-}=\lim _{\alpha \uparrow s} Z_{t}^{\alpha-}=M_{t}^{\prime} M_{s-}
$$

Here is a more particular situation.

Corollary 1 If $f \in C^{2}(\mathbb{R})$, we have for every $t \in T$

$$
\begin{aligned}
f\left(\tilde{N}_{t}\left(\tilde{N}_{1}-\tilde{N}_{t}\right)\right)= & f(0)+\left(\tilde{N}_{1}-\tilde{N}_{t}\right) \int_{0+}^{t} f^{\prime}\left(\tilde{N}_{s-}\left(\tilde{N}_{1}-\tilde{N}_{t}\right)\right) d \tilde{N}_{s} \\
& +\frac{1}{2}\left(\tilde{N}_{1}-\tilde{N}_{t}\right)^{2} \int_{0+}^{t} f^{\prime}\left(\tilde{N}_{s-}\left(\tilde{N}_{1}-\tilde{N}_{t}\right)\right) d s \\
& +\sum_{0 \leq s \leq t \leq 1}\left[f\left(\tilde{N}_{s}\left(\tilde{N}_{1}-\tilde{N}_{t}\right)\right)-f\left(\tilde{N}_{s-}\left(\tilde{N}_{1}-\tilde{N}_{t}\right)\right)\right. \\
& \left.-f^{\prime}\left(\tilde{N}_{s-}\left(\tilde{N}_{1}-\tilde{N}_{t}\right)\right)\left(\tilde{N}_{1}-\tilde{N}_{t}\right)\left(\tilde{N}_{s}-\tilde{N}_{s-}\right)\right] .
\end{aligned}
$$


Proof. Apply Proposition 5 with $M_{t}=\tilde{N}_{t}, M_{t}^{\prime}=\tilde{N}_{1}-\tilde{N}_{t}$ and $a \equiv 1$.

We conclude this section by proving a class of Burkholder type inequalities. These could an useful tool to bound the $L^{p}$-norms of anticipating integrals, since on the Poisson space there are no analogous of Meyer's inequalities (see [12]) proved for the operators $D$ and $\delta$ as defined in Section 2.

Proposition 6 (Burkholder inequalities) If $Y_{t}=\int_{0}^{t} \mathbf{E}\left(w_{s} \mid \mathbb{F}_{[s, t]^{c}}\right) d \tilde{N}_{s}$ with $w \in L^{2}(T \times \Omega)$, then, for every $p \geq 1$ there exist two universal constants $K_{1}(p)>0$ and $K_{2}(p)>0$ such that

$$
K_{1}(p) \mathbf{E}\left(\int_{0}^{t}\left(\mathbf{E}\left(w_{s} \mid \mathbb{F}_{[s, t]^{c}}\right)\right)^{2} d[\tilde{N}]_{s}\right)^{\frac{p}{2}} \leq \mathbb{E}\left|Y_{t}\right|^{p} \leq K_{2}(p) \mathbf{E}\left(\int_{0}^{t}\left(\mathbf{E}\left(w_{s} \mid \mathbb{F}_{[s, t]^{c}}\right)\right)^{2} d[\tilde{N}]_{s}\right)^{\frac{p}{2}}
$$

where $[\tilde{N}]_{t}=N_{t}$. In particular, let $X_{t}=\int_{0}^{t} u_{s} \delta \tilde{N}_{s}, t \in T$, where $u \in \mathbb{L}^{k, 2}, k \geq 3$, and let $w$ be the process defined in formula (25); then, for every $t \in T$,

$$
K_{1}(p) \mathbf{E}\left(\int_{0}^{t}\left(\mathbf{E}\left(w_{s} \mid \mathbb{F}_{[s, t]^{c}}\right)\right)^{2} d[\tilde{N}]_{s}\right)^{\frac{p}{2}} \leq \mathbf{E}\left|X_{t}\right|^{p} \leq K_{2}(p) \mathbf{E}\left(\int_{0}^{t}\left(\mathbf{E}\left(w_{s} \mid \mathbb{F}_{[s, t]^{c}}\right)\right)^{2} d[\tilde{N}]_{s}\right)^{\frac{p}{2}} .
$$

Proof. We have, by classical Burkholder inequalities for jump processes (see e.g. 223, Theorem 54])

$$
\begin{aligned}
\mathbf{E}\left|Y_{t}\right|^{p} & \leq \mathbf{E} \sup _{\lambda \leq t}\left|Y_{t}^{\lambda}\right|^{p} \\
& \leq K_{2}(p) \mathbf{E}\left(\int_{0}^{\lambda}\left(\mathbf{E}\left(w_{s} \mid \mathbb{F}_{[s, t]^{c}}\right)\right)^{2} d[\tilde{N}]_{s}\right)^{\frac{p}{2}} \\
& \leq K_{2}(p) \mathbf{E}\left(\int_{0}^{t}\left(\mathbf{E}\left(u_{s} \mid \mathbb{F}_{[s, t]^{c}}\right)\right)^{2} d[\tilde{N}]_{s}\right)^{\frac{p}{2}} .
\end{aligned}
$$

For the lower bound, we write

$$
\begin{aligned}
& \mathbf{E}\left|Y_{t}\right|^{p}=\mathbf{E} \lim _{\lambda \rightarrow t}\left|Y_{t}^{\lambda}\right|^{p}=\lim _{\lambda \rightarrow t} \mathbf{E}\left|Y_{t}^{\lambda}\right|^{p} \\
\geq & \lim _{\lambda \rightarrow t} K_{1}(p) \mathbf{E}\left(\int_{0}^{\lambda}\left(\mathbf{E}\left(w_{s} \mid \mathbb{F}_{[s, t]^{c}}\right)\right)^{2} d[\tilde{N}]_{s}\right)^{\frac{p}{2}}=\mathbf{E}\left(\int_{0}^{t}\left(\mathbf{E}\left(w_{s} \mid \mathbb{F}_{[s, t]^{c}}\right)\right)^{2} d[\tilde{N}]_{s}\right)^{\frac{p}{2}} .
\end{aligned}
$$

\section{References}

[1] K. Aase, B. Oksendhal, N. Privault and J. Uboe (2000) White noise generalizations of the ClarkHaussman-Ocone theorem and applications to mathematical finance. Finance and Stochastics, 4(4), 465-496.

[2] Abramowitz M. and Stegun I.A (1972) Handbook of Mathematical functions, New York, Dover. 
[3] E. Carlen and E. Pardoux (1990) Differential calculus and integration by parts on Poisson space. Stochastics, Algebra and Analysis in classical and quantum dynamics, 63-73, Kluwer.

[4] C. Dellacherie, B. Maissoneuve and P.A. Meyer (1992) Probabilités et Potentiel, Chapitres XVII à $X X I V$. Hermann.

[5] J. R. Elliott (1982) Stochastic Caluculs and Applications. Springer.

[6] I.I. Gihman and A.V. Skorohod (1979) The Theory of stochastic processes. Springer.

[7] Y. Kabanov (1975) On extended stochastic integrals. Theory of Probability and its applications, 20 710-722.

[8] I. Karatzas and S. Shreve (1991) Brownian motion and stochastic calculus. Springer.

[9] J.A. Leon and C. Tudor (1998) A chaos approach to the anticipating calculus for the Poisson process. Stochastics and Stochastics Reports, 62, 217-250.

[10] J. Ma, Ph. Protter and J. San Martin (1998). Anticipating integrals for a class of martingales. Bernoulli, 4, 81-114.

[11] N. Minh Duc and D. Nualart (1990) Stochastic processes possesing a Skorohod integral representation. Stochastics, 30(1), 47-60.

[12] D. Nualart (1995) Malliavin calculus and related topics. Springer.

[13] D. Nualart and E. Pardoux (1988) Stochastic calculus with anticipating integrands. Probability Theory and related Fields, 78, 535-581.

[14] D. Nualart and J. Vives (1990) Anticipative calculus for the Poisson space based on the Fock space. Séminaire de Probabilités XXIV, LNM 1426, Springer, 154-165.

[15] D. Nualart and M. Zakai (1998) Generalized multiple integrals and the representation of Wiener functionals. Stochastics, 23, 311-330.

[16] D. Ocone (1984) Malliavin's Calculus and Stochastic Integral Representation of Functionals of Diffusion Processes. Stochastics, 12, 161-185

[17] H. Ogura (1972) Orthogonal functionals of the Poisson process. IEEE Trans. Inform. Theory IT, 18, 473-481.

[18] G. Peccati, M. Thieullen and C.A. Tudor (2004) Martingale structure of Skorohod integral processes. To appear in The Annals of Probability.

[19] N. Privault, J.L. Solé and J. Vives (2000) Chaotic Kabanov formula for the Azéma martingales. Bernoulli 6(4), 633-651.

[20] N. Privault (1994) Chaotic and variational calculus in discrete and continuous time for the Poisson process. Stochastics and Stochastics Reports 51, 83-109.

[21] N. Privault (1994) Inégalités de Meyer sur l'espace de Poisson. C.R.A.S. 318, 559-562.

[22] N. Privault (1996) On the independence of multiple stochastic integrals with respect to a class of martingales. C.R.A.S. 323, 515-520. 
[23] Ph. Protter (1992) Stochastic integration and differential equations: a new approach. Springer.

[24] D. Revuz and M. Yor (1994) Continuous martingales and Brownian motion. Springer.

[25] A.V. Skorohod (1975) On a generalization of a stochastic integral. Theory of probability and its applications, 20, 219-233.

[26] C. Tudor (1997) Product formula for multiple Poisson-Itô integrals. Revue Roumaine de Math. Pures et Appliquées. 42(3-4), 339-345.

[27] C.A. Tudor and J. Vives (2002) The indefinite Skorohod integral as integrator on the Poisson space. Random Operators and Stochastic Equations.

[28] C.A. Tudor (2004) Martingale type stochastic calculus for anticipating integral processes. Bernoulli, 10(2), 313-325. 